\title{
Silver Nanowire-based Transparent Electrode as Flexible Anode for Large-Area Organic Light-Emitting Diodes
}

\author{
Minha Kim, Eun Jung, Sung Min Cho \\ School of Chemical Engineering, Sungkyunkwan University \\ 300 chunchun-dong, Jangan-gu, Suwon 440-746, Republic of Korea \\ min1004ha@naver.com; silverlayo@skku.edu; sungmcho@skku.edu
}

\section{Extended Abstract}

Silver nanowire-based transparent electrode was demonstrated as an anode for large-area organic light-emitting diodes (OLEDs). The electrode transparency depends on the density of the silver nanowire network that determines the sheet resistance of the electrode. The trade-off between the transparency and electrical resistance can be circumvented by utilizing an auxiliary silver-grid electrode for developing a large-area flexible electrode with high transparency and low resistance. In order to apply the silver nanowire-based transparent electrode to the anode for OLEDs, the surface roughness should be minimized to reduce the leakage current through the nanometer-thin devices. A roll-to-roll fabrication process was utilized to fabricate a large-area and planarized flexible transparent electrode with silver nanowire and silver auxiliary grid on a polyethylene terephthalate (PET) substrate. The sheet resistance and optical transmittance of the transparent electrode were $5 \mathrm{ohm} /$ square and $90 \%$, respectively. The planarized electrode surface was flat enough to fabricate OLED devices directly on the surface. Due to the scattering effect of the silver nanowire networks, the device efficiency was superior to that fabricated on the conventional ITO glass substrates.

Patterned large-area OLED devices were fabricated on the silver nanowire-based transparent anode using a roll-to-roll deposition process. The OLED devices is $10 \mathrm{~cm}$ wide and the length is not limited on a $15 \mathrm{~cm}$ wide PET roll. The OLED devices were protected with top and bottom moisture barrier layers deposited by an atomic layer deposition method from moisture and oxygen.[1,2] A few technical subjects on the roll-to-roll fabrication processes, emission uniformity, electrode uniformity and surface roughness will be discussed along with a few applications of the large-area flexible OLEDs in the presentation.

\section{References}

[1] S.-W. Seo, H. Chae, S. J. Seo, H. K. Chung, and S. M. Cho, Applied Physics Letters, vol. 102, 2013.

[2] S.-W. Seo, E. Jung, S. J. Seo, H. Chae, H. K. Chung, and S. M. Cho, Journal of Applied Physics, vol. 114, 2013. 